\title{
Bifurcation Structure of Equilibria of Iterated Softmax
}

\author{
Peter Tiňo \\ School of Computer Science \\ The University of Birmingham \\ Birmingham B15 2TT, UK \\ P.Tino@cs.bham.ac.uk
}

\begin{abstract}
We present a detailed bifurcation study of iterated renormalization process driven by softmax transformation parametrized by a temperature parameter. For each emerging equilibrium we give exact characterization of stable/unstable manifolds of the linearized dynamics. As the system cools down, new equilibria emerge in a strong structure until finally a complex skeleton of saddle type equilibria surrounding an unstable maximum entropy point, with decision enforcing "one-hot" stable equilibria emerges.
\end{abstract}

\section{Introduction}

There are situations where the quantities of interest, $w_{i} \in \mathbb{R}, i=1,2, \ldots, N$, need to be positive and sum up to one. In other words, the vector $\mathbf{w}=\left(w_{1}, w_{2}, \ldots, w_{N}\right)^{\prime}$ is constrained to the standard $(N-1)$-simplex

$$
S_{N-1}=\left\{\mathbf{w}=\left(w_{1}, w_{2}, \ldots, w_{N}\right)^{\prime} \in \mathbb{R}^{N} \mid w_{i} \geq 0, i=1,2, \ldots, N, \text { and } \sum_{i=1}^{N} w_{i}=1\right\} .
$$

For example, $w_{i}$ may be coefficients of a convex combination, or values of a multinomial distribution over $N$ outcomes ( 1 trial), or may represent 'soft' degrees of membership in an assignment problem.

One typical example of projecting onto $S_{N-1}$ is the softmax transformation ${ }^{1}$

$$
\mathbf{w} \mapsto \mathbf{F}(\mathbf{w} ; T)=\left(F_{1}(\mathbf{w} ; T), F_{2}(\mathbf{w} ; T), \ldots, F_{N}(\mathbf{w} ; T)\right)^{\prime},
$$

${ }^{1}$ related to Gibbs distribution formulation, or to the link function of multinomial distribution 
where $T>0$ is a (temperature) parameter,

$$
F_{i}(\mathbf{w} ; T)=\frac{\exp \left(\frac{w_{i}}{T}\right)}{Z(\mathbf{w} ; T)}, \quad i=1,2, \ldots, N
$$

and

$$
Z(\mathbf{w} ; T)=\sum_{k=1}^{N} \exp \left(\frac{w_{k}}{T}\right)
$$

is the normalization factor. For high and low settings of the temperature parameter $T$, the images under the softmax transformation cluster around the central point of $S_{N-1}$ and vertices of $S_{N-1}$, respectively.

Formally, the softmax transform $\mathbf{F}(2-4)$ maps $\mathbb{R}^{N}$ to $S_{N-1}^{0}$, the interior of $S_{N-1}$ :

$$
S_{N-1}^{0}=\left\{\mathbf{w} \in \mathbb{R}^{N} \mid w_{i}>0, i=1,2, \ldots, N, \text { and } \sum_{i=1}^{N} w_{i}=1\right\} .
$$

Linearization of $\mathbf{F}$ around $\mathbf{w} \in S_{N-1}^{0}$ is given by the (symmetric) Jacobian $J(\mathbf{w} ; T)$ :

$$
J(\mathbf{w} ; T)_{i, j}=\frac{1}{T}\left[\delta_{i, j} F_{i}(\mathbf{w} ; T)-F_{i}(\mathbf{w} ; T) F_{j}(\mathbf{w} ; T)\right], \quad i, j=1,2, \ldots, N,
$$

where $\delta_{i, j}=1$ iff $i=j$ and $\delta_{i, j}=0$ otherwise.

Typically, 'soft assignment' approaches to 'hard' 0-1 combinatorial assignment problems, or constrained optimization techniques searching over simplexes apply softmax renormalization repeatedly after each batch of assignment weight or parameter updates (see e.g. $[1,2,3,4,5])$. Iterated application of softmax transformation also appears e.g. in a posteriori corrections in classification machines [6].

Most 'soft assignment' optimization techniques employing periodic application of softmax transformation 'anneal' the solutions towards the 'hard' $0-1$ decisions by gradually decreasing the temperature parameter $T$. To have an insight about the effects of periodic or iterated application of the softmax transformation, it is useful to analyse the position and stability types of invariant sets of autonomous iterated softmax (ISM)

$$
\mathbf{w}(t+1)=\mathbf{F}(\mathbf{w}(t) ; T) \text {. }
$$

operating on on $S_{N-1}^{0}$. For example, by analyzing its first symmetry-breaking bifurcation point, we were able to find analytical expressions closely approximating critical temperatures for powerful intermittent search in a 'soft assignment' approach to solving $N$-queens problem [7]. In this paper we perform a detailed bifurcation study of the autonomous iterated softmax and give precise characterization and stability types of equilibria, as they 
emerge during the annealing process. Interesting and intricate equilibria structure emerges as the system cools down. At low temperatures, a complex skeleton of saddle type equilibria surrounding the unstable maximum entropy point (equal assignment weight for all assignment options), with decision enforcing "one-hot" stable equilibria (hard assignment decisions selecting exactly one assignment alternative) gradually emerges. This can lead to adaptation processes exhibiting signatures of complex dynamical behavior and sensitivity to annealing schedules, as empirically observed e.g. in [8].

It is worth noting that interesting bifurcation structure of fixed points have been revealed in other discrete or continuous time dynamical systems. For example, Agiza [9] studied fixed point stability of dynamics of 3 and 4 competitors in a Cournot game. Rich bifurcation structure has been identified with interesting pattern of periodic orbits emerging through Hopf bifurcation. Peng [10] showed how a stable closed invariant curve emerges from a fixed point in a two-dimensional delay population model via NeimarkSacker bifurcation. Codreanu and László [11] gave analytical underpinning to empirically observed fixed point bifurcations in some 1- and 2-dimensional iterative function systems. Fundamental role of symmetry-breaking bifurcation in a periodically driven planar pendulum is revealed in [12]. Sardanyés [13] illustrate how saddle-node bifurcation may play a key role in the information transfer in an error-prone self-replicating molecular system.

The paper has the following organization: We study the emerging structure and stability types of renormalization equilibria in sections 2 and 3, respectively. Key findings are summarized in section 4 . We will study systems for $N \geq 2$.

\section{Equilibria of Iterated Softmax}

First, we introduce basic concepts and notation that will be used throughout the paper.

In general, an $(n-1)$-simplex is just the convex hull of a set of $n$ affinely independent points in $\mathbb{R}^{m}, m \geq n-1$. A special case is the standard $(N-1)$-simplex $S_{N-1}$ defined in (1). It is the convex hull of the standard basis of $\mathbb{R}^{N},\left\{\mathbf{e}_{1}, \mathbf{e}_{2}, \ldots, \mathbf{e}_{N}\right\}$, where $\mathbf{e}_{i}$ is an $N$-dimensional vector of 0 's, except for the coordinate $i$ that has value 1 .

The convex hull of any nonempty subset of $n$ vertices of an $(r-1)$-simplex $\Delta, n \leq r$, is called an $(n-1)$-face of $\Delta$. There are $\left(\begin{array}{l}r \\ n\end{array}\right)$ distinct $(n-1)$-faces of $\Delta$. Each $(n-1)$-face is an $(n-1)$-simplex. In particular, the 0 -faces and 1 -faces are the vertices and edges, respectively, of $\Delta$. The $(r-2)$-faces are the facets of $\Delta$. Obviously, the unique $(r-1)$-face of $\Delta$ is the simplex $\Delta$ itself. 
Given a set of $n$ vertices $\mathbf{w}_{1}, \mathbf{w}_{2}, \ldots, \mathbf{w}_{n} \in \mathbb{R}^{m}$ defining an $(n-1)$-simplex $\Delta$ in $\mathbb{R}^{m}$, the central point,

$$
\overline{\mathbf{w}}(\Delta)=\frac{1}{n} \sum_{i=1}^{n} \mathbf{w}_{i}
$$

is called the maximum entropy point of $\Delta$.

We will denote the set of all $(n-1)$-faces of the standard $(N-1)$-simplex $S_{N-1}$ by $\mathcal{P}_{N, n}$. The set of their maximum entropy points is denoted by $\mathcal{Q}_{N, n}$, i.e.

$$
\mathcal{Q}_{N, n}=\left\{\overline{\mathbf{w}}(\Delta) \mid \Delta \in \mathcal{P}_{N, n}\right\}
$$

The $n$-dimensional column vectors of 1's and 0's are denoted by $\mathbf{1}_{n}$ and $\mathbf{0}_{n}$, respectively. Note that

$$
\overline{\mathbf{w}}_{N, n}=\frac{1}{n}\left(\mathbf{1}_{n}^{\prime}, \mathbf{0}_{N-n}^{\prime}\right)^{\prime}
$$

is in $\mathcal{Q}_{N, n}$. In addition, all the other elements of $\mathcal{Q}_{N, n}$ can be obtained by simply permuting coordinates of $\overline{\mathbf{w}}_{N, n}$. Due to this symmetry, we will be able to develop most of the material using $\overline{\mathbf{w}}_{N, n}$ only and then transfer the results to permutations of $\overline{\mathbf{w}}_{N, n}$. The maximum entropy point $\overline{\mathbf{w}}_{N, N}=N^{-1} \mathbf{1}_{N}$ of the standard $(N-1)$-simplex $S_{N-1}$ will often be denoted simply by $\overline{\mathbf{w}}$. To simplify the notation we will use $\overline{\mathbf{w}}$ to denote both the maximum entropy point of $S_{N-1}$ and the vector $\overline{\mathbf{w}}-\mathbf{0}_{N}$.

Let us now briefly recall findings of [7] that will be needed in this paper.

The maximum entropy point $\overline{\mathbf{w}}$ is a fixed point of ISM (7) for any temperature setting T. All the other fixed points $\mathbf{w}=\left(w_{1}, w_{2}, \ldots, w_{N}\right)^{\prime}$ of ISM have exactly two different coordinate values: $w_{i} \in\left\{\gamma_{1}, \gamma_{2}\right\}$, such that $N^{-1}<\gamma_{1}<N_{1}^{-1}$ and $0<\gamma_{2}<N^{-1}$, where $N_{1}$ is the number of coordinates $\gamma_{1}$ larger than $N^{-1}$. Since $\mathbf{w} \in S_{N-1}^{0}$, we have

$$
\gamma_{2}=\frac{1-N_{1} \gamma_{1}}{N-N_{1}}
$$

The number of coordinates $\gamma_{2}$ smaller than $N^{-1}$ is denoted by $N_{2}$. Obviously, $N_{2}=N-N_{1}$.

If $\mathbf{w}=\left(\gamma_{1} \mathbf{1}_{N_{1}}^{\prime}, \gamma_{2} \mathbf{1}_{N_{2}}^{\prime}\right)^{\prime}$ is a fixed point of ISM (7), so are all $\left(\begin{array}{c}N \\ N_{1}\end{array}\right)$ distinct permutations of it. We collect $\mathbf{w}$ and its permutations in a set defined as follows: given integers $N \geq 2$, $0<N_{1}<N$, and a real number $N^{-1}<\gamma_{1}<N_{1}^{-1}$, we write

$$
\mathcal{E}_{N, N_{1}}\left(\gamma_{1}\right)=\left\{\mathbf{v} \in S_{N-1}^{0} \mid \mathbf{v} \text { is a permutation of }\left(\gamma_{1} \mathbf{1}_{N_{1}}^{\prime}, \frac{1-N_{1} \gamma_{1}}{N-N_{1}} \mathbf{1}_{N-N_{1}}^{\prime}\right)^{\prime}\right\} .
$$

The fixed points in $\mathcal{E}_{N, N_{1}}\left(\gamma_{1}\right)$ exist if and only if the temperature parameter $T$ is set to

$$
T_{N, N_{1}}\left(\gamma_{1}\right)=\left(N \gamma_{1}-1\right)\left[-\left(N-N_{1}\right) \cdot \ln \left(1-\frac{N \gamma_{1}-1}{\left(N-N_{1}\right) \gamma_{1}}\right)\right]^{-1} \text {. }
$$


For a given $N \geq 2$ and $0<N_{1}<N$, the temperature function $T_{N, N_{1}}\left(\gamma_{1}\right):\left(N^{-1}, N_{1}^{-1}\right) \rightarrow$ $\mathbb{R}^{+}$is concave and

$$
\lim _{\gamma_{1} \rightarrow N^{-1}} T_{N, N_{1}}\left(\gamma_{1}\right)=N^{-1}
$$

In addition, the slope of $T_{N, N_{1}}\left(\gamma_{1}\right)$ at $N^{-1}$ approaches

$$
\kappa_{N, N_{1}}=\lim _{\gamma_{1} \rightarrow N^{-1}} \frac{\mathrm{d} T_{N, N_{1}}\left(\gamma_{1}\right)}{\mathrm{d} \gamma_{1}}=1-\frac{N}{2\left(N-N_{1}\right)} .
$$

For temperatures $T>1 / 2$, the maximum entropy point $\overline{\mathbf{w}}$ is the unique equilibrium of ISM.

\subsection{Bifurcations of equilibria of ISM}

In this section we examine the mechanism of emergence of new ISM equilibria, as the system anneals. As mentioned earlier, the maximum entropy point $\overline{\mathbf{w}}=N^{-1} \mathbf{1}_{N}$ is a fixed point of ISM (7) for any temperature setting. The existence of other fixed points depends on the temperature $T$, as described by (13). We will show that as the system cools down, increasing number of equilibria emerge in a strong structure.

Let $\mathbf{w}, \mathbf{v} \in S_{N-1}$ be two points on the standard simplex. The line from $\mathbf{w}$ to $\mathbf{v}$ is parametrized as

$$
\ell(\tau ; \mathbf{w}, \mathbf{v})=\mathbf{w}+\tau \cdot(\mathbf{v}-\mathbf{w}), \quad \tau \in[0,1]
$$

Theorem 2.1 All equilibria of ISM (7) lie on lines connecting the maximum entropy point $\overline{\mathbf{w}}$ of $S_{N-1}$ with the maximum entropy points of its faces. In particular, for $0<N_{1}<N$ and $N^{-1}<\gamma_{1}<N_{1}^{-1}$, all fixed points from $\mathcal{E}_{N, N_{1}}\left(\gamma_{1}\right)$ lie on lines $\ell(\tau ; \overline{\mathbf{w}}, \mathbf{w})$, where $\mathbf{w} \in \mathcal{Q}_{N, N_{1}}$.

Proof: We know that for a given $0<N_{1}<N$ and $N^{-1}<\gamma_{1}<N_{1}^{-1}$, there is a temperature setting $T_{N, N_{1}}\left(\gamma_{1}\right)$ such that all permutations of $\left(\gamma_{1} \mathbf{1}_{N_{1}}^{\prime}, \gamma_{2} \mathbf{1}_{N_{2}}^{\prime}\right)^{\prime}$, where $N_{2}=$ $N-N_{1}$ and $\gamma_{2}$ is given by (11), are fixed points of ISM. Instead of dealing with the whole set $\mathcal{E}_{N, N_{1}}\left(\gamma_{1}\right)$, we will concentrate only on the representative $\mathbf{w}\left(\gamma_{1}\right)=\left(\gamma_{1} \mathbf{1}_{N_{1}}^{\prime}, \gamma_{2} \mathbf{1}_{N_{2}}^{\prime}\right)^{\prime}$; the result then follows by symmetry.

Consider the maximum entropy point $\overline{\mathbf{w}}_{N, N_{1}}$ of an $\left(N_{1}-1\right)$-face of $S_{N-1}$ (see eq. (10)), $\overline{\mathbf{w}}_{N, N_{1}}=\frac{1}{N_{1}}\left(\mathbf{1}_{N_{1}}^{\prime}, \mathbf{0}_{N_{2}}^{\prime}\right)^{\prime}$. Then $\mathbf{w}\left(\gamma_{1}\right)$ lies on the line $\ell\left(\tau ; \overline{\mathbf{w}}, \overline{\mathbf{w}}_{N, N_{1}}\right)$ for the parameter 
setting $\tau=1-N \gamma_{2}$. In other words,

$$
\begin{aligned}
& \gamma_{1}=\frac{1}{N}\left(1+\tau \frac{N_{2}}{N 1}\right) \\
& \gamma_{2}=\frac{1}{N}(1-\tau),
\end{aligned}
$$

which can be easily verified by plugging in $\tau=1-N \gamma_{2}$ into (17).

For an ISM fixed point $\mathbf{w} \in \mathcal{E}_{N, N_{1}}\left(\gamma_{1}\right)$ we will denote by $\Gamma(\mathbf{w})$ the maximum entropy point $\Gamma(\mathbf{w}) \in \mathcal{Q}_{N, N_{1}}$ of the $\left(N_{1}-1\right)$-face of $S_{N-1}$, such that $\mathbf{w} \in \ell(\tau ; \overline{\mathbf{w}}, \Gamma(\mathbf{w}))$.

The result is illustrated in figure 1 . Fixed points of ISM operating on the standard 3 -simplex $S_{3}$ can only be found on the lines connecting the maximum entropy point $\overline{\mathbf{w}}$ (filled circle) with maximum entropy points of its faces. Triangles, squares and diamonds represent maximum entropy points of 0 -faces (vertices), 1-faces (edges) and 2-faces (facets), respectively. Fixed points with three coordinates larger than $1 / 4$ can be be found on the lines connecting $\overline{\mathbf{w}}$ with maximum entropy points of 2 -faces (solid bold lines). Analogously, fixed points with two and one coordinate larger than $1 / 4$ can be be found on the lines connecting $\overline{\mathbf{w}}$ with maximum entropy points of 1- and 0-faces (dashed and dashed-dotted bold lines, respectively).

Theorem 2.2 For $N_{1}<N / 2$, there exists a temperature $T_{E}\left(N, N_{1}\right)>N^{-1}$ such that for $T \in\left(0, T_{E}\left(N, N_{1}\right)\right]$, ISM fixed points in $\mathcal{E}_{N, N_{1}}\left(\gamma_{1}\right)$ exist for some $\gamma_{1} \in\left(N^{-1}, N_{1}^{-1}\right)$, and no ISM fixed points in $\mathcal{E}_{N, N_{1}}\left(\gamma_{1}\right)$, for any $\gamma_{1} \in\left(N^{-1}, N_{1}^{-1}\right)$, can exist at temperatures $T>T_{E}\left(N, N_{1}\right)$. The temperature $T_{E}\left(N, N_{1}\right)$ is given by $T_{N, N_{1}}\left(\gamma_{1}^{*}\right)$, where $\gamma_{1}^{*}$ is the unique solution of

$$
\ln \left[\frac{\left(N-N_{1}\right) \gamma_{1}}{1-N_{1} \gamma_{1}}\right]=\frac{\gamma_{1}-\frac{1}{N}}{\gamma_{1}\left(1-N_{1} \gamma_{1}\right)} .
$$

For each temperature $T \in\left(N^{-1}, T_{E}\left(N, N_{1}\right)\right)$, there are two coordinate values $\gamma_{1}^{-}(T)$ and $\gamma_{1}^{+}(T), N^{-1}<\gamma_{1}^{-}(T)<\gamma_{1}^{+}(T)<N_{1}^{-1}$, such that ISM fixed points in both $\mathcal{E}_{N, N_{1}}\left(\gamma_{1}^{-}(T)\right)$ and $\mathcal{E}_{N, N_{1}}\left(\gamma_{1}^{+}(T)\right)$ exist at temperature $T$. Furthermore, as the temperature decreases, $\gamma_{1}^{-}(T)$ decreases towards $N^{-1}$, while $\gamma_{1}^{+}(T)$ increases towards $N_{1}^{-1}$.

Proof: The temperature function $T_{N, N_{1}}\left(\gamma_{1}\right)(13)$ is concave and can be continuously extended to $\left[N^{-1}, N_{1}^{-1}\right)$. At $\gamma_{1}=N^{-1}$, the temperature is $N^{-1}$ and since $N_{1}<N / 2$, the slope of $T_{N, N_{1}}\left(\gamma_{1}\right)$ at $\gamma_{1}=N^{-1}$ is positive. Because

$$
\lim _{\gamma_{1} \rightarrow N_{1}^{-1}} T_{N, N_{1}}\left(\gamma_{1}\right)=0<N^{-1}
$$




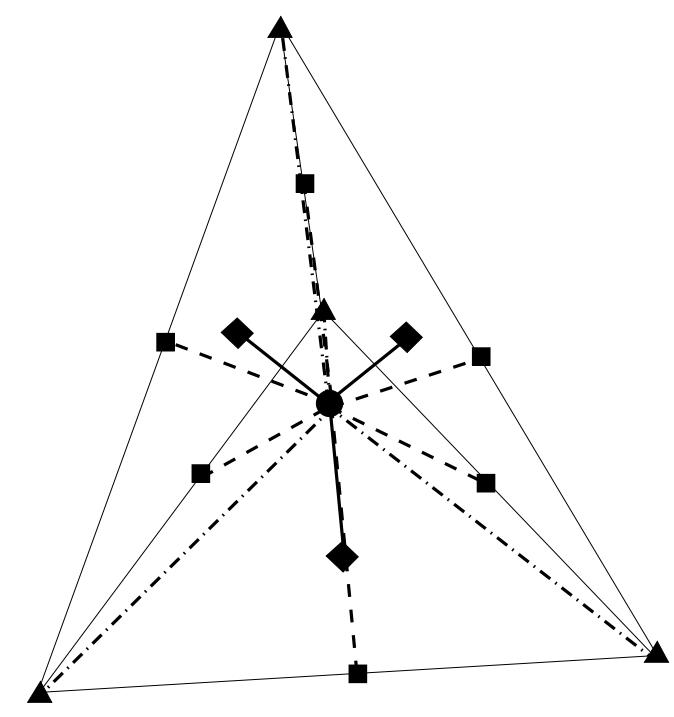

Figure 1: Positions of ISM equilibria illustrated for the case of 4-dimensional assignment weight vectors $\mathbf{w}$. ISM is operating on the standard 3 -simplex $S_{3}$ and its equilibria can only be found on the lines connecting the maximum entropy point $\overline{\mathbf{w}}$ (filled circle) with maximum entropy points of its faces. Triangles, squares and diamonds represent maximum entropy points of 0-faces (vertices), 1-faces (edges) and 2-faces (facets), respectively.

$T_{N, N_{1}}\left(\gamma_{1}\right)$ must have a unique maximum at some $\gamma_{1}^{*} \in\left(N^{-1}, N_{1}^{-1}\right)$,

$$
\gamma_{1}^{*}=\underset{\gamma_{1} \in\left(N^{-1}, N_{1}^{-1}\right)}{\operatorname{argmax}} T_{N, N_{1}}\left(\gamma_{1}\right) .
$$

Setting the derivative of $T_{N, N_{1}}\left(\gamma_{1}\right)$ to zero leads to (19).

Obviously, no ISM fixed points in $\mathcal{E}_{N, N_{1}}\left(\gamma_{1}\right)$, for any $N^{-1}<\gamma_{1}<N_{1}^{-1}$, can exist for temperatures greater than $T_{N, N_{1}}\left(\gamma_{1}^{*}\right)$.

Since $T_{N, N_{1}}\left(\gamma_{1}\right)$ is concave, for temperatures $N^{-1}<T<T_{N, N_{1}}\left(\gamma_{1}^{*}\right)$, there are two coordinate values $\gamma_{1}^{-}(T)$ and $\gamma_{1}^{+}(T), \gamma_{1}^{-}(T)<\gamma_{1}^{+}(T)$, such that $T=T_{N, N_{1}}\left(\gamma_{1}^{-}(T)\right)=$ $T_{N, N_{1}}\left(\gamma_{1}^{+}(T)\right)$. The values $\gamma_{1}^{-}(T)$ and $\gamma_{1}+(T)$ correspond to the increasing and decreasing branches of $T_{N, N_{1}}\left(\gamma_{1}\right)$, and so as the temperature decreases, $\gamma_{1}^{-}(T)$ decreases as well, while $\gamma_{1}^{+}(T)$ increases.

Note that for low-temperature regimes, $T<N^{-1}$, there is only one value of $\gamma_{1}(T)$ close to $N_{1}^{-1}$, such that $T=T_{N, N_{1}}\left(\gamma_{1}(T)\right)$. In other words, if $N_{1}<N / 2$, in low temperature regimes the fixed points tend to accumulate around the maximum entropy points of $\left(N_{1}-\right.$ 1)-faces of $S_{N-1}$. The same is true for the ISM fixed points with $N_{1} \geq N / 2$ coordinates $\gamma_{1}$ larger than $N^{-1}$. 
Theorem 2.3 If $N / 2 \leq N_{1}<N$, for each temperature $T \in\left(0, N^{-1}\right)$, there is exactly one coordinate value $\gamma_{1}(T) \in\left(N^{-1}, N_{1}^{-1}\right)$, such that ISM fixed points in $\mathcal{E}_{N, N_{1}}\left(\gamma_{1}(T)\right)$ exist at temperature T. No ISM fixed points in $\mathcal{E}_{N, N_{1}}\left(\gamma_{1}\right)$, for any $\gamma_{1} \in\left(N^{-1}, N_{1}^{-1}\right)$ can exist for temperatures $T>N^{-1}$. As the temperature decreases, $\gamma_{1}(T)$ increases towards $N_{1}^{-1}$.

Proof: $\quad$ Since $N / 2 \leq N_{1}<N$, the slope of $T_{N, N_{1}}\left(\gamma_{1}\right)$ at $\gamma_{1}=N^{-1}$ is not positive. In addition, $T_{N, N_{1}}\left(\gamma_{1}\right)$ is concave. It follows that for temperatures $T>N^{-1}$, there is no $\gamma_{1}(T)$ such that $T=T_{N, N_{1}}\left(\gamma_{1}(T)\right)$. For $0<T<N^{-1}, T_{N, N_{1}}\left(\gamma_{1}\right)$ is decreasing and there is exactly one $\gamma_{1}(T)$ such that $T=T_{N, N_{1}}\left(\gamma_{1}(T)\right)$.

Next, we will show that the set of bifurcation temperatures $T_{E}\left(N, N_{1}\right)>N^{-1}$, for $N_{1}<N / 2$, is linearly ordered.

Lemma 2.4 Consider $0<N_{1}<N_{1}^{\prime}<N$ and a coordinate value $\gamma_{1} \in\left(1 / N, 1 / N_{1}^{\prime}\right)$. Then,

$$
\gamma_{2}=\frac{1-N_{1} \gamma_{1}}{N-N_{1}}>\frac{1-N_{1}^{\prime} \gamma_{1}}{N-N_{1}^{\prime}}=\gamma_{2}^{\prime} .
$$

In addition, $T_{N, N_{1}^{\prime}}\left(\gamma_{1}\right)<T_{N, N_{1}}\left(\gamma_{1}\right)$.

Proof: $\quad$ Let $N_{1}^{\prime}=N_{1}+1$ and assume

$$
\gamma_{2}=\frac{1-N_{1} \gamma_{1}}{N-N_{1}} \leq \frac{1-\left(N_{1}+1\right) \gamma_{1}}{N-N_{1}-1}=\gamma_{2}^{\prime}
$$

Then,

$$
\left(N-N_{1}\right)\left(1-N_{1} \gamma_{1}-\gamma_{1}\right) \geq\left(N-N_{1}-1\right)\left(1-N_{1} \gamma_{1}\right),
$$

which is equivalent to $\gamma_{1} \leq N^{-1}$. This is a contradiction. Hence, $\gamma_{2}>\gamma_{2}^{\prime}$. If $N_{1}^{\prime}=N_{1}+k$, for some $k>1,(20)$ can be obtained by induction.

Note that the temperature function can be re-written as

$$
T_{N, N_{1}}\left(\gamma_{1}\right)=\frac{\gamma_{1}-\gamma_{2}}{\ln \gamma_{1}-\ln \gamma_{2}}
$$

with $\gamma_{2}$ given by (11).

Logarithm is a concave function. Since $0<\gamma_{2}^{\prime}<\gamma_{2}<\gamma_{1}<1$, we have

$$
T_{N, N_{1}}\left(\gamma_{1}\right)=\frac{\gamma_{1}-\gamma_{2}}{\ln \gamma_{1}-\ln \gamma_{2}}>\frac{\gamma_{1}-\gamma_{2}^{\prime}}{\ln \gamma_{1}-\ln \gamma_{2}^{\prime}}=T_{N, N_{1}^{\prime}}\left(\gamma_{1}\right)
$$


Theorem 2.5 The bifurcation temperature $T_{E}\left(N, N_{1}\right)$ is decreasing with increasing number $N_{1}$ of equilibrium coordinates larger than $N^{-1}$.

Proof: $\quad$ By lemma 2.4, for any feasible value of the larger coordinate $\gamma_{1}>N^{-1}$, if there are two fixed points $\mathbf{w} \in \mathcal{E}_{N, N_{1}}\left(\gamma_{1}\right)$ and $\mathbf{w}^{\prime} \in \mathcal{E}_{N, N_{1}^{\prime}}\left(\gamma_{1}\right)$ of ISM, such that $N_{1}<N_{1}^{\prime}$, then $\mathbf{w}$ exists at a higher temperature than $\mathbf{w}^{\prime}$ does.

For a given $N_{1}<N / 2$, the bifurcation temperature $T_{E}\left(N, N_{1}\right)$ corresponds to the maximum of $T_{N, N_{1}}\left(\gamma_{1}\right)$ on $\gamma_{1} \in\left(N^{-1}, N_{1}^{-1}\right)$. It follows that $N_{1}<N_{1}^{\prime}$ implies $T_{E}\left(N, N_{1}\right)>$ $T_{E}\left(N, N_{1}^{\prime}\right)$.

We now summarize the results of this section. For temperatures $T>1 / 2$, the ISM has exactly one equilibrium - the maximum entropy point $\overline{\mathbf{w}}$ of $S_{N-1}$. As the temperature is lowered and hits the first bifurcation point, $T_{E}(N, 1)$, new fixed points of ISM emerge on the lines $\ell(\tau ; \overline{\mathbf{w}}, \widetilde{\mathbf{w}}), \widetilde{\mathbf{w}} \in \mathcal{Q}_{N, 1}$, one on each line. The lines connect $\overline{\mathbf{w}}$ with the vertices $\widetilde{\mathbf{w}}$ of $S_{N-1}$. As the temperature decreases further, on each line, the single fixed point splits into two fixed points, one moves towards $\overline{\mathbf{w}}$, the other moves towards the corresponding high entropy point $\widetilde{\mathbf{w}}$ in $\mathcal{Q}_{N, 1}$ (vertex of $S_{N-1}$ ). When the temperature reaches the second bifurcation point, $T_{E}(N, 2)$, new fixed points of ISM emerge on the lines $\ell(\tau ; \overline{\mathbf{w}}, \widetilde{\mathbf{w}}), \widetilde{\mathbf{w}} \in \mathcal{Q}_{N, 2}$, one on each line. This time the lines connect $\overline{\mathbf{w}}$ with the maximum entropy points (midpoints) $\widetilde{\mathbf{w}}$ of the edges of $S_{N-1}$. Again, as the temperature continues decreasing, on each line, the single fixed point splits into two fixed points, one moves towards $\overline{\mathbf{w}}$, the other moves towards the corresponding maximum entropy point $\widetilde{\mathbf{w}}$ in $\mathcal{Q}_{N, 2}$ (midpoint of an edge of $S_{N-1}$ ). Decreasing temperature further to $T_{E}(N, 3)$, new fixed points of ISM emerge on the lines $\ell(\tau ; \overline{\mathbf{w}}, \widetilde{\mathbf{w}}), \widetilde{\mathbf{w}} \in \mathcal{Q}_{N, 3}$, connecting $\overline{\mathbf{w}}$ with maximum entropy points $\widetilde{\mathbf{w}}$ of 2 -faces of $S_{N-1}$. Decreasing the temperature even further, on each line, the single fixed point splits into two fixed points, one moves towards $\overline{\mathbf{w}}$, the other moves towards the corresponding maximum entropy point $\widetilde{\mathbf{w}}$ in $\mathcal{Q}_{N, 3}$. The process continues until the last bifurcation temperature $T_{E}\left(N, N_{1}\right)$ is reached, where $N_{1}$ is the largest natural number smaller than $N / 2$. At $T_{E}\left(N, N_{1}\right)$, new fixed points of ISM emerge on the lines $\ell(\tau ; \overline{\mathbf{w}}, \widetilde{\mathbf{w}}), \widetilde{\mathbf{w}} \in \mathcal{Q}_{N, N_{1}}$, connecting $\overline{\mathbf{w}}$ with maximum entropy points $\widetilde{\mathbf{w}}$ of $\left(N_{1}-1\right)$-faces of $S_{N-1}$. As the temperature continues decreasing, on each line, the single fixed point splits into two fixed points, one moves towards $\overline{\mathbf{w}}$, the other moves towards the corresponding maximum entropy point $\widetilde{\mathbf{w}}$ in $\mathcal{Q}_{N, N_{1}}$. At temperatures below $N^{-1}$, only the fixed points moving towards the maximum entropy points of faces of $S_{N-1}$ exist. 


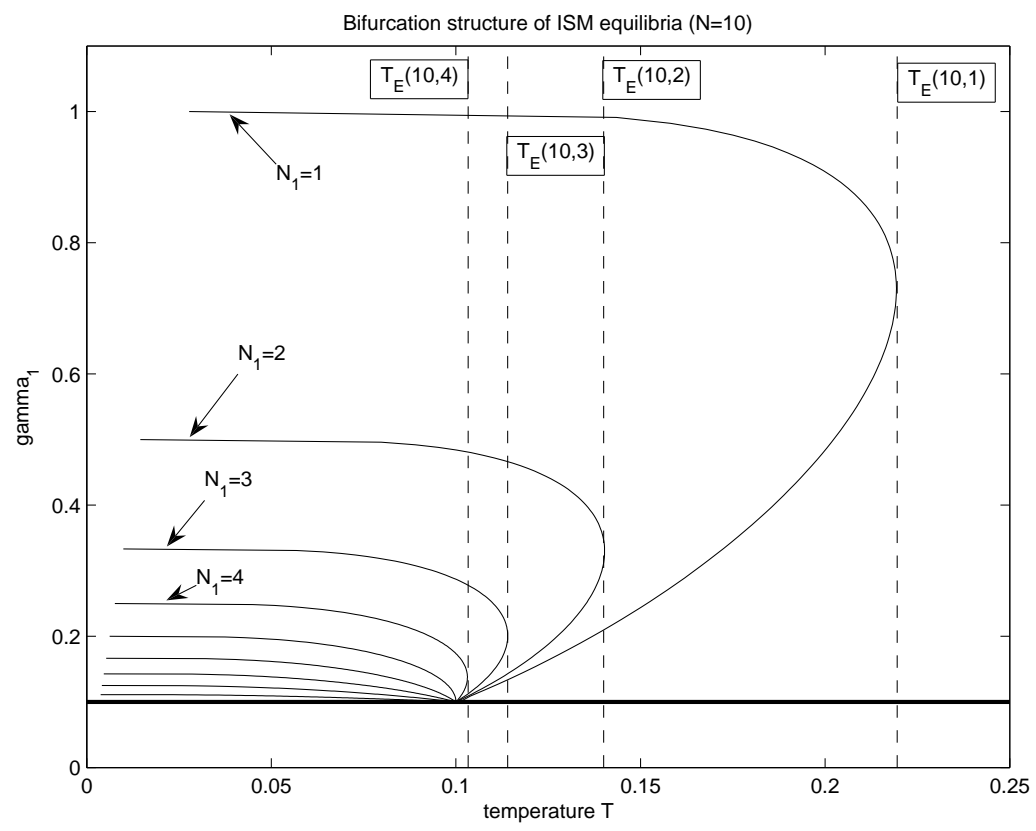

Figure 2: Demonstration of the process of creation of new ISM fixed points and their flow as the system temperature cools down. Here $N=10$, e.g. the ISM operates on the standard 9-simplex $S_{9}$. Against each temperature setting $T$, the values of the larger coordinate $\gamma_{1}>N^{-1}$ of the fixed points existing at $T$ are plotted. The horizontal bold line corresponds to the maximum entropy point $\overline{\mathbf{w}}=10^{-1} \mathbf{1}_{10}^{\prime}$.

In the low temperature regime, $0<T<N^{-1}$, a fixed point occurs on every line $\ell(\tau ; \overline{\mathbf{w}}, \widetilde{\mathbf{w}}), \widetilde{\mathbf{w}} \in \mathcal{Q}_{N, N_{1}}, N_{1}=\lceil N / 2\rceil,\lceil N / 2\rceil+1, \ldots, N-1$. Here, $\lceil x\rceil$ denotes the smallest integer $y$, such that $y \geq x$. As the temperature decreases, the fixed points $\mathbf{w}$ move towards the corresponding maximum entropy points $\Gamma(\mathbf{w})=\widetilde{\mathbf{w}} \in \mathcal{Q}_{N, N_{1}}$ of $\left(N_{1}-1\right)$-faces of $S_{N-1}$.

The process of creation of new fixed points and their flow as the temperature cools down is demonstrated in figure 2 for an ISM operating on 9 -simplex $S_{9}$. We plot against each temperature setting $T$ the values of the larger coordinate $\gamma_{1}>N^{-1}=0.1$ of the fixed points existing at $T$.

Next theorem summarizes the number of ISM equilibria as a function of temperature $T$.

Theorem 2.6 At temperature $T>0$ there exist $1+\mathcal{N}_{N}(T)$ fixed points of ISM (7), where

1. for temperatures $T>T_{E}(N, 1), \mathcal{N}_{N}(T)=0$, 
2. for temperatures $T=T_{E}\left(N, N_{1}\right), N_{1}=1,2, \ldots,\lceil N / 2\rceil-1$,

$$
\mathcal{N}_{N}(T)=\left(\begin{array}{c}
N \\
N_{1}
\end{array}\right)+2 \sum_{n=1}^{N_{1}-1}\left(\begin{array}{l}
N \\
n
\end{array}\right),
$$

3. for temperatures $T_{E}\left(N, N_{1}\right)<T<T_{E}\left(N, N_{1}-1\right), N_{1}=2, \ldots,\lceil N / 2\rceil-1$,

$$
\mathcal{N}_{N}(T)=2 \sum_{n=1}^{N_{1}-1}\left(\begin{array}{l}
N \\
n
\end{array}\right)
$$

4. for temperatures $N^{-1}<T<T_{E}(N,\lceil N / 2\rceil-1)$,

$$
\mathcal{N}_{N}(T)=2 \sum_{n=1}^{\lceil N / 2\rceil-1}\left(\begin{array}{l}
N \\
n
\end{array}\right),
$$

5. at temperature $T=N^{-1}$,

$$
\mathcal{N}_{N}(T)=\sum_{n=1}^{\lceil N / 2\rceil-1}\left(\begin{array}{l}
N \\
n
\end{array}\right),
$$

6. for temperatures $0<T<N^{-1}$,

$$
\mathcal{N}_{N}(T)=\sum_{n=1}^{N-1}\left(\begin{array}{l}
N \\
n
\end{array}\right) .
$$

Proof: The result follows from the ISM bifurcation scenario outlined above and realizing that there are $\left(\begin{array}{l}N \\ n\end{array}\right)(n-1)$-faces in an $(N-1)$-simplex.

1. For $T>T_{E}(N, 1)$ there is a unique fixed point $\overline{\mathbf{w}} \in S_{N-1}$ of ISM (7).

2. When $T=T_{E}\left(N, N_{1}\right), N_{1}=1,2, \ldots,\lceil N / 2\rceil-1,\left(\begin{array}{c}N \\ N_{1}\end{array}\right)$ ISM fixed points are created on $\left(\begin{array}{c}N \\ N_{1}\end{array}\right)$ lines from $\overline{\mathbf{w}}$ to $\left(N_{1}-1\right)$-faces of $S_{N-1}$. For each $1 \leq n<N_{1}$, there already exists a pair of ISM fixed points on each of $\left(\begin{array}{l}N \\ n\end{array}\right)$ lines from $\overline{\mathbf{w}}$ to $(n-1)$-faces of $S_{N-1}$. The maximum entropy fixed point $\overline{\mathbf{w}}$ of ISM (7) always exists.

3. For $T_{E}\left(N, N_{1}\right)<T<T_{E}\left(N, N_{1}-1\right), N_{1}=2, \ldots,\lceil N / 2\rceil-1$, for each $1 \leq n<N_{1}$, there exists a pair of ISM fixed points on each of $\left(\begin{array}{l}N \\ n\end{array}\right)$ lines from $\overline{\mathbf{w}}$ to $(n-1)$-faces of $S_{N-1}$.

4. For $N^{-1}<T<T_{E}(N,\lceil N / 2\rceil-1)$, all "paired" ISM fixed points exist, and so for each $1 \leq n<\lceil N / 2\rceil$, there is a pair of ISM fixed points on each of $\left(\begin{array}{l}N \\ n\end{array}\right)$ lines from $\overline{\mathbf{w}}$ to $(n-1)$-faces of $S_{N-1}$. 
5. At $T=N^{-1}$, for every $1 \leq n<\lceil N / 2\rceil$, the ISM fixed points closer to $\overline{\mathbf{w}}$ on each of $\left(\begin{array}{l}N \\ n\end{array}\right)$ lines from $\overline{\mathbf{w}}$ to $(n-1)$-faces of $S_{N-1}$ seize to exist and those lines now contain a single ISM fixed point each.

6. For $0<T<N^{-1}$, a single ISM fixed point on each of the lines connecting $\overline{\mathbf{w}}$ with each face ${ }^{2}$ of $S_{N-1}$ exists.

Having characterized the number and position of ISM equilibria, in the next section we present their stability analysis.

\section{Stability analysis of renormalization equilibria}

The maximum entropy point $\overline{\mathbf{w}}$ is not only a fixed point of ISM (7), but also, regarded as a vector $\overline{\mathbf{w}}-\mathbf{0}_{N}$, it is an eigenvector of the Jacobian $J(\mathbf{w} ; T)$ (see eq. (6)) at any $\mathbf{w} \in S_{N-1}^{0}$, with eigenvalue $\lambda=0$. This simply reflects the fact that ISM renormalization acts on the standard simplex $S_{N-1}$, which is a subset of a $(N-1)$-dimensional hyperplane with normal vector $\mathbf{1}_{N}$.

We have already seen that the maximum entropy point $\overline{\mathbf{w}}$ plays a special role in the ISM equilibria structure: all equilibria lie on lines going from $\overline{\mathbf{w}}$ towards maximum entropy points of faces of $S_{N-1}$. The lines themselves are of special interest, since we will show that these lines are invariant manifolds of the ISM renormalization and their directional vectors are eigenvectors of ISM Jacobians at the fixed points located on them.

First, we need an auxiliary lemma.

Lemma 3.1 Let $\mathbf{w} \in \mathcal{E}_{N, N_{1}}\left(\gamma_{1}\right)$ for some $1 \leq N_{1}<N, \gamma_{1} \in\left(N^{-1}, N_{1}^{-1}\right)$, be a fixed point of ISM (7) with squared Euclidean norm $\|\mathbf{w}\|^{2}=\mathbf{w}^{\prime} \mathbf{w}$. Then, $\gamma_{1}>\|\mathbf{w}\|^{2}$ and $\gamma_{2}<\|\mathbf{w}\|^{2}$, where $\gamma_{2}=\left(1-N_{1} \gamma_{1}\right) /\left(N-N_{1}\right)$.

Proof: We have

$$
\|\mathbf{w}\|^{2}=N_{1} \gamma_{1}^{2}+N_{2} \gamma_{2}^{2}=\frac{N N_{1} \gamma_{1}^{2}-2 N_{1} \gamma_{1}+1}{N-N_{1}} .
$$

\footnotetext{
${ }^{2}$ except for $S_{N-1}$ itself
} 
Now, $\|\mathbf{w}\|^{2}-\gamma_{1}<0$ only if

$$
\kappa\left(\gamma_{1}\right)=N N_{1} \gamma_{1}^{2}-\gamma_{1}\left(N+N_{1}\right)+1<0 .
$$

Given $N, N_{1}, \kappa\left(\gamma_{1}\right)$ can be considered quadratic function of $\gamma_{1}$ on $\mathbb{R}$ with zeros at $\gamma_{1}=N^{-1}$ and $\gamma_{1}=N_{1}^{-1}$. Hence $\kappa\left(\gamma_{1}\right)<0$ for all $\gamma_{1}$ in $\left(N^{-1}, N_{1}^{-1}\right)$, exactly the allowed range for $\gamma_{1}$.

The statement $\gamma_{2}<\|\mathbf{w}\|^{2}$ can be proved in a completely symmetric manner.

Theorem 3.2 Let $\mathbf{w} \in \mathcal{E}_{N, N_{1}}\left(\gamma_{1}\right)$ for some $1 \leq N_{1}<N, \gamma_{1} \in\left(N^{-1}, N_{1}^{-1}\right)$, be a fixed point of ISM (7). Then, $\mathbf{w}_{*}=\overline{\mathbf{w}}-\mathbf{w}$ is an eigenvector of the Jacobian $J\left(\mathbf{w} ; T_{N, N_{1}}\left(\gamma_{1}\right)\right)$ of ISM at $\mathbf{w}$ with the corresponding eigenvalue

$$
\lambda_{*}=\frac{\gamma_{1} \cdot\left(\gamma_{1}-\|\mathbf{w}\|^{2}\right)}{T_{N, N_{1}}\left(\gamma_{1}\right) \cdot\left(\gamma_{1}-N^{-1}\right)}>0,
$$

where $\|\mathbf{w}\|^{2}=\mathbf{w}^{\prime} \mathbf{w}$ is the squared Euclidean norm of $\mathbf{w}$.

Proof: To simplify the notation, we will denote the Jacobian of ISM at $\mathbf{w}=$ $\left(w_{1}, w_{2}, \ldots, w_{N}\right)^{\prime}$ by $J$ and the temperature at which $\mathbf{w}$ exists by $T$. From

$$
J \mathbf{w}_{*}=J(\overline{\mathbf{w}}-\mathbf{w})=J \overline{\mathbf{w}}-J \mathbf{w}=-J \mathbf{w},
$$

and using (6), we have that the $i$-th element of $J \mathbf{w}_{*}$ is equal to

$$
\frac{w_{i}}{T} \mathbf{w}^{\prime} \mathbf{w}-w_{i} \mathbf{e}_{i}^{\prime} \mathbf{w}=\frac{w_{i}}{T}\left(\|\mathbf{w}\|^{2}-w_{i}\right)
$$

For $\mathbf{w}_{*}$ to be an eigenvector of $J$ with eigenvalue $\lambda_{*}$, it must hold

$$
J \mathbf{w}_{*}=\lambda_{*} \overline{\mathbf{w}}-\lambda_{*} \mathbf{w},
$$

and so the $i$-th element of $J \mathbf{w}_{*}$ must also be equal to $\lambda_{*} N^{-1}-\lambda_{*} w_{i}$. Hence,

$$
\frac{w_{i}}{T}\left(\|\mathbf{w}\|^{2}-w_{i}\right)=\lambda_{*} N^{-1}-\lambda_{*} w_{i}
$$

should hold for all $i=1,2, \ldots, N$. But we know that $w_{i} \in\left\{\gamma_{1}, \gamma_{2}\right\}, \gamma_{2}=\left(1-N_{1} \gamma_{1}\right) /(N-$ $N_{1}$ ), and so

$$
\frac{\gamma_{1} \cdot\left(\gamma_{1}-\|\mathbf{w}\|^{2}\right)}{\gamma_{2} \cdot\left(\gamma_{2}-\|\mathbf{w}\|^{2}\right)}=\frac{\gamma_{1}-N^{-1}}{\gamma_{2}-N^{-1}}
$$


would need to be true. Since $\gamma_{1}, \gamma_{2}>0,(25)$ can be rewritten as

$$
\frac{\gamma_{1}-\|\mathbf{w}\|^{2}}{\gamma_{2}-\|\mathbf{w}\|^{2}}=\frac{1-\left(N \gamma_{1}\right)^{-1}}{1-\left(N \gamma_{2}\right)^{-1}}
$$

To verify whether (26) holds, we write

$$
\begin{aligned}
\frac{\gamma_{1}-\|\mathbf{w}\|^{2}}{\gamma_{2}-\|\mathbf{w}\|^{2}} & =\frac{\gamma_{1}-\|\mathbf{w}\|^{2}}{\frac{1-N_{1} \gamma_{1}}{N-N_{1}}-\|\mathbf{w}\|^{2}} \\
& =1-\frac{1-N \gamma_{1}}{1-N_{1} \gamma_{1}-\left(N-N_{1}\right)\|\mathbf{w}\|^{2}}
\end{aligned}
$$

and

$$
\begin{aligned}
\frac{1-\frac{1}{N \gamma_{1}}}{1-\frac{1}{N \gamma_{2}}} & =\frac{\frac{N \gamma_{1}-1}{N \gamma_{1}}}{\frac{N_{1}\left(N \gamma_{1}-1\right)}{N\left(N_{1} \gamma_{1}-1\right)}} \\
& =1-\frac{1}{N_{1} \gamma_{1}} .
\end{aligned}
$$

We need to show that

$$
\frac{1-N \gamma_{1}}{1-N_{1} \gamma_{1}-\left(N-N_{1}\right)\|\mathbf{w}\|^{2}}=\frac{1}{N_{1} \gamma_{1}} .
$$

Note that the denominators in (29) are guaranteed to be nonzero, since $N_{1}, \gamma_{1}>0$ and by lemma 3.1,

$$
1-N_{1} \gamma_{1}-\left(N-N_{1}\right)\|\mathbf{w}\|^{2}=1-N_{1} \gamma_{1}-N_{2}\|\mathbf{w}\|^{2}<1-N_{1} \gamma_{1}-N_{2} \gamma_{2}=0 .
$$

Hence, (29) can be restated as

$$
N_{1} \gamma_{1}\left(1-N \gamma_{1}\right)=1-N_{1} \gamma_{1}-\left(N-N_{1}\right)\|\mathbf{w}\|^{2} .
$$

But (30) holds, since

$$
\|\mathbf{w}\|^{2}=N_{1} \gamma_{1}^{2}+N_{2} \gamma_{2}^{2}=N_{1} \gamma_{1}^{2}+\frac{\left(1-N_{1} \gamma_{1}\right)^{2}}{N-N_{1}}
$$

The expression (23) for $\lambda_{*}$ follows from (24), by plugging $\operatorname{in}^{3} \gamma_{1}$ for $w_{i}$.

It remains to be shown that $\lambda_{*}$ is positive. This follows directly from (23) since $\gamma_{1}>N^{-1}>0, T>0$, and by lemma $3.1, \gamma_{1}-\|\mathbf{w}\|^{2}>0$.

Theorem 3.3 Let $\mathbf{w} \in \mathcal{E}_{N, N_{1}}\left(\gamma_{1}\right)$ for some $\lceil N / 2\rceil \leq N_{1} \leq N-1, \gamma_{1} \in\left(N^{-1}, N_{1}^{-1}\right)$, be a fixed point of ISM (7) and $\lambda_{*}$ be the eigenvalue associated with eigenvector $\mathbf{w}_{*}=\overline{\mathbf{w}}-\mathbf{w}$ of the Jacobian $J\left(\mathbf{w} ; T_{N, N_{1}}\left(\gamma_{1}\right)\right)$ of ISM at $\mathbf{w}$. Then, $0<\lambda_{*}<1$.

\footnotetext{
3 alternatively, we could have used $\gamma_{2}$
} 


\section{Proof:}

Using parametrization (17-18),

$$
\begin{aligned}
\|\mathbf{w}\|^{2} & =N_{1} \gamma_{1}^{2}+N_{2} \gamma_{2}^{2} \\
& =\frac{1}{N}\left(1+\tau^{2} \frac{N_{2}}{N_{1}}\right)
\end{aligned}
$$

so that

$$
\begin{aligned}
\gamma_{1}-\|\mathbf{w}\|^{2} & =\frac{1}{N}\left(\tau \frac{N_{2}}{N_{1}}-\tau^{2} \frac{N_{2}}{N_{1}}\right) \\
& =\frac{N_{2}}{N_{1} N} \tau(1-\tau)
\end{aligned}
$$

and since

$$
\gamma_{1}-N^{-1}=\frac{N_{2}}{N_{1} N} \tau,
$$

we can write

$$
\frac{\gamma_{1}-\|\mathbf{w}\|^{2}}{\gamma_{1}-N^{-1}}=1-\tau .
$$

From (23) and (34) we obtain

$$
\lambda_{*}=\frac{\gamma_{1}}{T}(1-\tau) .
$$

Now, assume $\lambda_{*} \geq 1$. That means (using (35) and (18))

$$
\frac{T}{\gamma_{1}}=\frac{1-\frac{\gamma_{2}}{\gamma_{1}}}{-\ln \frac{\gamma_{2}}{\gamma_{1}}} \leq 1-\tau=\gamma_{1} N \frac{\gamma_{2}}{\gamma_{1}},
$$

which can be written as

$$
\frac{a-1}{\ln a} \leq N \gamma_{1} a
$$

where

$$
0<a=\frac{\gamma_{2}}{\gamma_{1}}<1
$$

Since

$$
N_{1} \gamma_{1}+\left(N-N_{1}\right) \gamma_{2}=N_{1} \gamma_{1}+\left(N-N_{1}\right) a \gamma_{1}=1
$$

we have

$$
\gamma_{1}=\left(N_{1}+\left(N-N_{1}\right) a\right)^{-1}
$$


and (36) reads ${ }^{4}$

$$
\begin{aligned}
\ln a & \leq-\frac{1-a}{a}(\rho+a(1-\rho)) \\
& =-\rho \frac{(1-a)^{2}}{a}+a-1=f(a ; \rho) .
\end{aligned}
$$

where

$$
0<\rho=\frac{N_{1}}{N}<1 .
$$

The function $f(a ; \rho)$ is increasing on $a \in(0,1)$ (actually on $a>0$ ), since its derivative $f^{\prime}(a)=\rho / a^{2}+1-\rho$ is positive. The second derivative of $f, f^{\prime \prime}(a)=-2 \rho / a^{3}$, is negative for $a>0$, and so $f(a)$ is concave.

On $a>0$, both $\ln a$ and $f(a)$ are continuous concave functions with $\ln 1=f(1)=0$ and $\ln ^{\prime}(1)=f^{\prime}(1)=1$. So the function values, as well as the slopes of $\ln a$ and $f(a)$ coincide at $a=1$. We will investigate conditions under which the slope of $f(a)$ exceeds that of $\ln a$ on the whole interval $(0,1)$. Then, for $a \in(0,1)$, we would have $f(a)<\ln a$.

The inequality

$$
f^{\prime}(a)-\ln ^{\prime}(a)>0
$$

holds, whenever $1-\rho+\rho a^{-2}-a^{-1}>0$, which is equivalent to

$$
g(a)=a^{2}(1-\rho)-a+\rho>0 .
$$

Since $1-\rho>0, g(a)>0$ on $a \in\left(0, a_{1}\right) \cup\left(a_{2}, 1\right)$, where $a_{1}, a_{2}$ are zeros of the quadratic function $g(a)$,

$$
a_{1,2}=\frac{1 \pm|1-2 \rho|}{2(1-\rho)} .
$$

For $\rho \in(0,1 / 2)$, we have $a_{1}=\rho /(1-\rho)$ and $a_{2}=1$, whereas for $\rho \in[1 / 2,1), a_{1}=1$ and $a_{2}=\rho /(1-\rho)$. The boundary points $a_{1}$ and $a_{2}$, plotted as functions of $\rho \in(0,1)$, are shown in figure 3 .

Because $N_{1} \geq\lceil N / 2\rceil$, we have $\rho \geq 1 / 2$, and so $g(a)>0$ on $a \in(0,1)$. But $a=\gamma_{2} / \gamma_{1}$ is always in the interval $(0,1)$ (see eq. (37)). It follows that $f(a)<\ln a$ for any $a \in(0,1)$. This is a contradiction to (38), and so it must be that $\lambda_{*}<1$. By theorem $3.2, \lambda_{*}$ is positive.

\footnotetext{
${ }^{4} \ln a<0$
} 


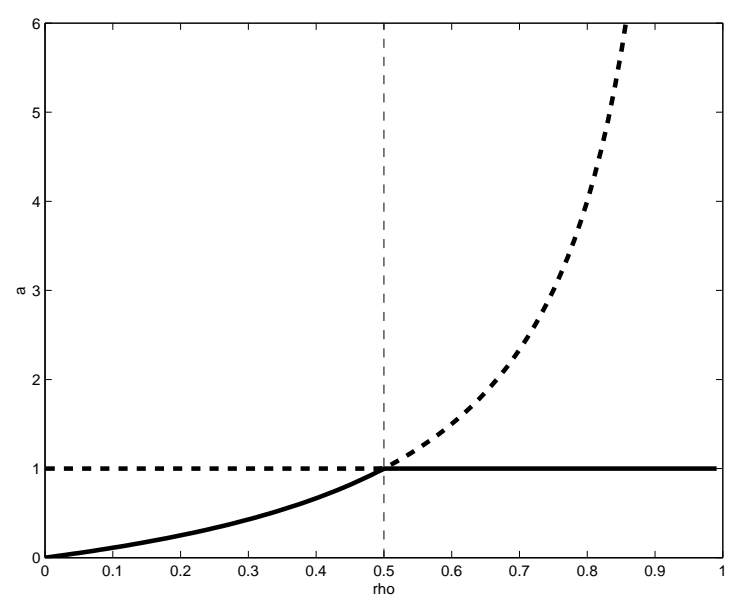

Figure 3: Boundary points $a_{1}$ (solid bold line) and $a_{2}$ (dashed bold line), plotted as functions of $\rho \in(0,1)$. The quadratic function $g(a)$ (see (40)) is positive for $a<a_{1}$ and $a>a_{2}$.

Theorem 3.4 Let $\mathbf{w} \in \mathcal{E}_{N, N_{1}}\left(\gamma_{1}\right)$ for some $1 \leq N_{1}<\lceil N / 2\rceil$ and $N^{-1}<\gamma_{1}<\left(2 N_{1}\right)^{-1}$, be a fixed point of ISM (7) and $\lambda_{*}$ be the eigenvalue associated with eigenvector $\mathbf{w}_{*}=\overline{\mathbf{w}}-\mathbf{w}$ of the Jacobian $J\left(\mathbf{w} ; T_{N, N_{1}}\left(\gamma_{1}\right)\right)$ of ISM at $\mathbf{w}$. Then, $\lambda_{*}>1$.

Proof: First note that the bound $N^{-1}<\gamma_{1}<\left(2 N_{1}\right)^{-1}$ is well defined, since from $N_{1}<N / 2$ we have $1 /\left(2 N_{1}\right)>1 / N$.

The proof proceeds analogously to the proof of theorem 3.3, but this time we are interested in the negative range of $g(a)$, implying $f^{\prime}(a)<\ln ^{\prime}(a)$. For $a \in(0,1), g(a)<0$ only on $a \in\left(a_{1}, 1\right)$. Since $\ln a$ and $f(a)$ are continuous concave functions with $\ln 1=f(1)=$ $0, \ln ^{\prime}(1)=f^{\prime}(1)=1$, and $f^{\prime}(a)<\ln ^{\prime}(a)$, it must be that on $a \in\left(a_{1}, 1\right), f(a)>\ln a$. That means $\lambda_{*}>1$.

For $\rho \in(0,1 / 2)$,

$$
a_{1}=\frac{\rho}{1-\rho}=\frac{\frac{N_{1}}{N}}{\frac{N-N_{1}}{N}}=\frac{N_{1}}{N_{2}}
$$

Now,

$$
\frac{1-N_{1} \gamma_{1}}{N_{2} \gamma_{1}}=\frac{\gamma_{2}}{\gamma_{1}}=a>a_{1}=\frac{N_{1}}{N_{2}}
$$

only if $\gamma_{1}<1 /\left(2 N_{1}\right)$. 
Theorem 3.5 Consider ISM (7) and $1 \leq N_{1}<\lceil N / 2\rceil$. Then there exists $\bar{\gamma}_{1} \in\left(\left(2 N_{1}\right)^{-1}, N_{1}^{-1}\right)$, such that for all ISM fixed points $\mathbf{w} \in \mathcal{E}_{N, N_{1}}\left(\gamma_{1}\right)$ with $\gamma_{1} \in\left(\bar{\gamma}_{1}, N_{1}^{-1}\right)$, the eigenvalue associated with eigenvector $\mathbf{w}_{*}=\overline{\mathbf{w}}-\mathbf{w}$ of the Jacobian $J\left(\mathbf{w} ; T_{N, N_{1}}\left(\gamma_{1}\right)\right)$ of ISM at $\mathbf{w}$ is positive and less than 1, e.g. $0<\lambda_{*}<1$.

Proof: $\quad$ Based on proof of theorem 3.3, for $a \in\left(0, a_{1}\right)$, we have $f^{\prime}(a)>\ln ^{\prime}(a)$. This corresponds to $\gamma_{1}>1 /\left(2 N_{1}\right)$ (see proof of theorem 3.4).

Now, $\ln a$ and $f(a)$ are continuous concave functions with $\ln 1=f(1)=0, \ln ^{\prime}(1)=$ $f^{\prime}(1)=1$, and on $a \in\left(a_{1}, 1\right)$, we have $f^{\prime}(a)<\ln ^{\prime}(a)$, implying that for $a \in\left(a_{1}, 1\right)$, $\ln a<f(a)$.

Since

$$
\lim _{a \rightarrow 0^{+}} f(a)=\lim _{a \rightarrow 0^{+}} \ln a=\infty
$$

and (using L'Hospital rule)

$$
\lim _{a \rightarrow 0^{+}} \frac{\ln a}{f(a)}=0
$$

there exists $\bar{a} \in\left(0, a_{1}\right)$, such that on $a \in(0, \bar{a}), f(a)<\ln a$ (both $f(a)$ and $\ln a$ are negative). The coordinate $\bar{\gamma}_{1}$ corresponds to the value $\bar{a}$. Since $\bar{a}<a_{1}$, we have $\bar{\gamma}_{1}>$ $1 /\left(2 N_{1}\right)$. Furthermore, $a \in(0, \bar{a})$ corresponds to $\gamma_{1} \in\left(\bar{\gamma}_{1}, N_{1}^{-1}\right)$.

Theorem 3.6 Consider ISM (7) and $1 \leq N_{1}<N$. Then for each maximum entropy point $\widetilde{\mathbf{w}} \in \mathcal{Q}_{N, N_{1}}$ of an $\left(N_{1}-1\right)$-face of $S_{N-1}$, the line $\ell(\tau ; \overline{\mathbf{w}}, \widetilde{\mathbf{w}}), t \in[0,1)$ (eqs. (1718)), connecting the maximum entropy point $\overline{\mathbf{w}}$ with $\widetilde{\mathbf{w}}$ is an invariant set under the ISM dynamics.

Proof: Without loss of generality, consider the canonical form of $\widetilde{\mathbf{w}} \in \mathcal{Q}_{N, N_{1}}$, namely

$$
\widetilde{\mathbf{w}}=\overline{\mathbf{w}}_{N, N_{1}}=\frac{1}{N_{1}}\left(\mathbf{1}_{N_{1}}^{\prime}, \mathbf{0}_{N-N_{1}}^{\prime}\right)^{\prime},
$$

a parameter value $\tau \in[0,1)$ and the point $\mathbf{w}(\tau)=\ell(\tau ; \overline{\mathbf{w}}, \widetilde{\mathbf{w}})$ addressed by it on the line connecting $\overline{\mathbf{w}}$ with $\widetilde{\mathbf{w}} \in \mathcal{Q}_{N, N_{1}}$. The image $\mathbf{F}(\mathbf{w}(\tau))$ of $\mathbf{w}(\tau)$ under the ISM has the following form:

$$
\gamma_{1}=F_{i}(\mathbf{w}(\tau))=\frac{\exp \left\{\frac{1}{T N}(1-\tau)+\frac{1}{T N_{1}} \tau\right\}}{N_{1} \exp \left\{\frac{1}{T N}(1-\tau)+\frac{1}{T N_{1}} \tau\right\}+N_{2} \exp \left\{\frac{1}{T N}(1-\tau)\right\}}
$$




$$
=\frac{\exp \left\{\frac{1}{T N_{1}} \tau\right\}}{N_{1} \exp \left\{\frac{1}{T N_{1}} \tau\right\}+N_{2}}, \quad i=1,2, \ldots, N_{1},
$$

and

$$
\begin{aligned}
\gamma_{2}=F_{i}(\mathbf{w}(\tau)) & =\frac{\exp \left\{\frac{1}{T N}(1-\tau)\right\}}{N_{1} \exp \left\{\frac{1}{T N}(1-\tau)+\frac{1}{T N_{1}} \tau\right\}+N_{2} \exp \left\{\frac{1}{T N}(1-\tau)\right\}} \\
& =\frac{1}{N_{1} \exp \left\{\frac{1}{T N_{1}} \tau\right\}+N_{2}}, \quad i=N_{1}+1, N_{1}+2, \ldots, N
\end{aligned}
$$

Denoting $N_{1} \exp \left\{\frac{1}{T N_{1}} \tau\right\}+N_{2}$ by $Z$, the parameter value $\tau$ giving $\gamma_{2}=Z^{-1}$ under the line parametrization (18) is

$$
\tau^{*}=1-\frac{N}{Z} .
$$

Plugging $\tau^{*}$ into the parametrization (17) for $\gamma_{1}$ yields

$$
\frac{Z+N_{1}-N}{Z N_{1}}=\frac{Z-N_{2}}{Z N_{1}}=\frac{\exp \left\{\frac{1}{T N_{1}} \tau\right\}}{Z} .
$$

So the image under ISM map $\mathbf{F}$ of any point $\mathbf{w}(\tau)=\ell(\tau ; \overline{\mathbf{w}}, \widetilde{\mathbf{w}}), \tau \in[0.1)$, can be written as $\mathbf{F}(\mathbf{w}(\tau))=\ell\left(\tau^{*} ; \overline{\mathbf{w}}, \widetilde{\mathbf{w}}\right)$, where $0 \leq \tau^{*}=1-N / Z<1$.

We have established that for any fixed point $\mathbf{w} \in S_{N-1}^{0}$ of ISM (7), $\mathbf{w}_{*}=\overline{\mathbf{w}}-\mathbf{w}$ is always an eigendirection of the linearized system at $\mathbf{w}$. If $\mathbf{w} \in \mathcal{E}_{N, N_{1}}\left(\gamma_{1}\right)$ for some $\lceil N / 2\rceil \leq N_{1}<N, \gamma_{1} \in\left(N^{-1}, N_{1}^{-1}\right)$, the eigendirection $\mathbf{w}_{*}$ is stable with non-oscillatory behavior, i.e. the associated eigenvalue $\lambda_{*}$ of the ISM Jacobian at $\mathbf{w}$ is a real positive number, smaller than 1 . If $1 \leq N_{1}<\lceil N / 2\rceil$, the eigendirection $\mathbf{w}_{*}$ is stable for fixed points close enough to the maximum entropy points of faces of $S_{N-1}$, for those close to $\overline{\mathbf{w}}$, the eigendirection $\mathbf{w}_{*}$ is unstable. Moreover, $\mathbf{1}_{N}$ is always an eigendirection of the linearized ISM system with zero eigenvalue. As mentioned earlier, this is due to the fact that ISM renormalization acts on the standard simplex $S_{N-1}$, which is part of an $(N-1)$-dimensional hyperplane with normal vector $\mathbf{1}_{N}$.

We now continue with analysis of the remaining $N-2$ eigendirections of the linearized ISM system at its fixed points $\mathbf{w}$. Recall that $\Gamma(\mathbf{w}) \in \mathcal{Q}_{N, N_{1}}$ denotes the maximum entropy point of an $\left(N_{1}-1\right)$-face of $S_{N-1}$, such that $\mathbf{w}$ lies on the line segment connecting $\overline{\mathbf{w}}$ with $\Gamma(\mathbf{w})$.

Theorem 3.7 Consider a ISM fixed point $\mathbf{w} \in \mathcal{E}_{N, N_{1}}\left(\gamma_{1}\right)$ for some $1 \leq N_{1}<N$ and $N^{-1}<\gamma_{1}<N_{1}^{-1}$. Let $\pi$ be a permutation of $\overline{\mathbf{w}}_{N, N_{1}}=\frac{1}{N_{1}}\left(\mathbf{1}_{N_{1}}^{\prime}, \mathbf{0}_{N-N_{1}}^{\prime}\right)^{\prime}$ such that 
$\pi\left(\overline{\mathbf{w}}_{N, N_{1}}\right)=\Gamma(\mathbf{w}) . \quad$ Let $\mathcal{B}=\left\{\mathbf{u}_{1}, \mathbf{u}_{2}, \ldots, \mathbf{u}_{N-N_{1}-1}\right\}$ be a set of $\left(N-N_{1}\right)$-dimensional unit vectors, such that $\mathcal{B}$, together with $\mathbf{1}_{N-N_{1}} /\left\|\mathbf{1}_{N-N_{1}}\right\|$, form an orthonormal basis of $\mathbb{R}^{N-N_{1}}$. Then, there are $N-N_{1}-1$ eigenvectors of the Jacobian $J\left(\mathbf{w} ; T_{N, N_{1}}\left(\gamma_{1}\right)\right)$ of ISM at $\mathbf{w}$ of the form:

$$
\mathbf{v}_{-}^{i}=\pi\left(\left(\mathbf{0}_{N_{1}}^{\prime}, \mathbf{u}_{i}^{\prime}\right)^{\prime}\right), \quad i=1,2, \ldots, N-N_{1}-1 .
$$

All eigenvectors $\mathbf{v}_{-}^{1}, \mathbf{v}_{-}^{2}, \ldots, \mathbf{v}_{-}^{N-N_{1}-1}$ have the same associated eigenvalue

$$
0<\lambda_{-}=\frac{1-N_{1} \gamma_{1}}{\left(N-N_{1}\right) T_{N, N_{1}}\left(\gamma_{1}\right)}<1
$$

Proof: Without loss of generality assume that $\mathbf{w}=\left(\gamma_{1} \mathbf{1}_{N_{1}}^{\prime}, \gamma_{2} \mathbf{1}_{N_{2}}^{\prime}\right)^{\prime}$, where $\gamma_{2}$ is defined by (11) and $N_{2}=N-N_{1}$. All the results translate to permutations of $\mathbf{w}$ in a straightforward manner.

To simplify the notation, we will denote the Jacobian $J\left(\mathbf{w} ; T_{N, N_{1}}\left(\gamma_{1}\right)\right)$ by $J$ and the temperature $T_{N, N_{1}}\left(\gamma_{1}\right)$ at which $\mathbf{w}$ exists by $T$. The $n$-dimensional identity matrix is denoted by $I_{n}$.

The Jacobian can be written as

$$
J=\frac{-1}{T}\left[\begin{array}{cc}
G_{1} & G_{12} \\
G_{12}^{\prime} & G_{2}
\end{array}\right],
$$

where $G_{1}$ is an $N_{1} \times N_{1}$ symmetric matrix

$$
G_{1}=\gamma_{1}\left(\gamma_{1} \mathbf{1}_{N_{1}} \mathbf{1}_{N_{1}}^{\prime}-I_{N_{1}}\right)
$$

$G_{2}$ is an $N_{2} \times N_{2}$ symmetric matrix

$$
G_{2}=\gamma_{2}\left(\gamma_{2} \mathbf{1}_{N_{2}} \mathbf{1}_{N_{2}}^{\prime}-I_{N_{2}}\right)
$$

and $G_{12}$ is an $N_{1} \times N_{2}$ matrix

$$
G_{12}=\gamma_{1} \gamma_{2} \mathbf{1}_{N_{1}} \mathbf{1}_{N_{2}}^{\prime}
$$

For $\mathbf{u}=\left(u_{1}, u_{2}, \ldots, u_{N_{2}}\right)^{\prime} \in \mathcal{B}$

$$
\left[\begin{array}{c}
G_{12} \\
G_{2}
\end{array}\right] \mathbf{u}=\left[\begin{array}{c}
\gamma_{1} \gamma_{2} \Sigma(\mathbf{u}) \mathbf{1}_{N_{1}} \\
\gamma_{2}^{2} \Sigma(\mathbf{u}) \mathbf{1}_{N_{2}}-\gamma_{2} \mathbf{u}
\end{array}\right],
$$

where $\Sigma(\mathbf{u})=\sum_{i=1}^{N_{2}} u_{i}$. All $\mathbf{u} \in \mathcal{B}$ are orthogonal to $\mathbf{1}_{N_{2}}$, implying that $\Sigma(\mathbf{u})=0$. Hence,

$$
\left[\begin{array}{c}
G_{12} \\
G_{2}
\end{array}\right] \mathbf{u}=-\gamma_{2}\left[\begin{array}{c}
\mathbf{0}_{N_{1}} \\
\mathbf{u}
\end{array}\right] .
$$


It follows that for all $i=1,2, \ldots, N-N_{1}-1$,

$$
J \mathbf{v}_{-}^{i}=\frac{\gamma_{2}}{T} \mathbf{v}_{-}^{i},
$$

meaning that $\mathbf{v}_{-}^{i}$ is an eigenvector of $J$ with eigenvalue $\lambda_{-}=\gamma_{2} / T$. Now, $\lambda_{-}>0$, since $\gamma_{2}>0$ and $T>0$. It remains to be shown that $\lambda_{-}<1$.

By $(21)$,

$$
\lambda_{-}=\frac{\ln \frac{\gamma_{1}}{\gamma_{2}}}{\frac{\gamma_{1}}{\gamma_{2}}-1}=\frac{\ln b}{b-1},
$$

where $b=\gamma_{1} / \gamma_{2}>1$. But $0<\ln b<b-1$ on $b \in(1, \infty)$ and so $\lambda_{-}<1$.

Theorem 3.8 Consider a ISM fixed point $\mathbf{w} \in \mathcal{E}_{N, N_{1}}\left(\gamma_{1}\right)$ for some $1 \leq N_{1}<N$ and $N^{-1}<\gamma_{1}<N_{1}^{-1}$. Let $\pi$ be a permutation of $\overline{\mathbf{w}}_{N, N_{1}}=\frac{1}{N_{1}}\left(\mathbf{1}_{N_{1}}^{\prime}, \mathbf{0}_{N-N_{1}}^{\prime}\right)^{\prime}$ such that $\pi\left(\overline{\mathbf{w}}_{N, N_{1}}\right)=\Gamma(\mathbf{w})$. Let $\mathcal{B}=\left\{\mathbf{u}_{1}, \mathbf{u}_{2}, \ldots, \mathbf{u}_{N_{1}-1}\right\}$ be a set of $N_{1}$-dimensional unit vectors, such that $\mathcal{B}$, together with $\mathbf{1}_{N_{1}} /\left\|\mathbf{1}_{N_{1}}\right\|$, form an orthonormal basis of $\mathbb{R}^{N_{1}}$. Then, there are $N_{1}-1$ eigenvectors of the Jacobian $J\left(\mathbf{w} ; T_{N, N_{1}}\left(\gamma_{1}\right)\right)$ of ISM at $\mathbf{w}$ of the form:

$$
\mathbf{v}_{+}^{i}=\pi\left(\left(\mathbf{u}_{i}^{\prime}, \mathbf{0}_{N-N_{1}}^{\prime}\right)^{\prime}\right), \quad i=1,2, \ldots, N_{1}-1 .
$$

All eigenvectors $\mathbf{v}_{+}^{1}, \mathbf{v}_{+}^{2}, \ldots, \mathbf{v}_{+}^{N_{1}-1}$ have the same associated eigenvalue

$$
\lambda_{+}=\frac{\gamma_{1}}{T_{N, N_{1}}\left(\gamma_{1}\right)}>1
$$

Proof: $\quad$ Using arguments analogous to those in the proof of theorem 3.7 one obtains that for all $i=1,2, \ldots, N_{1}-1$,

$$
J \mathbf{v}_{+}^{i}=\frac{\gamma_{1}}{T} \mathbf{v}_{+}^{i}
$$

By $(21)$,

$$
\lambda_{+}=\frac{\ln \frac{\gamma_{2}}{\gamma_{1}}}{\frac{\gamma_{2}}{\gamma_{1}}-1}=\frac{\ln a}{a-1},
$$

where $0<a=\gamma_{2} / \gamma_{1}<1$. By noting that on $a \in(0,1), \ln a<a-1<0$, we get $\lambda_{+}>1$.

\section{Conclusion}

We have rigorously analyzed bifurcations of equilibria of iterated softmax (ISM) renormalization. As the system cools down, new ISM equilibria emerge on the lines connecting 
the maximum entropy point $\overline{\mathbf{w}}$ of the standard simplex $S_{N-1}$ with the maximum entropy points of its faces of increasing dimensionality. Depending on the face dimensionality, one or two fixed points can exist on each line. The dimensionality of stable and unstable manifolds of linearized ISM at emerging equilibria decreases and increases, respectively as the temperature decreases. At low temperatures, a complex skeleton of saddle type equilibria surrounding the unstable maximum entropy point $\overline{\mathbf{w}}$ (representing equal assignment weight for all assignment options), with decision enforcing "one-hot" stable equilibria located near vertices of $S_{N-1}$ (representing hard assignment decisions selecting exactly one assignment alternative) gradually emerges. In "soft assignment" optimization techniques employing periodic application of the softmax transformation while annealing the solutions towards the "hard" 0-1 decisions, such a bifurcation structure may lead to adaptation processes exhibiting signatures of complex dynamical behavior and high sensitivity to annealing schedules, as empirically observed e.g. in [8]. While the present study provides clues for understanding potential complications in annealing to optimal solutions in soft assignment optimization approaches employing soft-max renormalization, a complete understanding can only be achieved by studying the full optimization dynamics in such systems, with assignment adaptations iteratively coupled with soft-max projections onto the solution simplex. This is a matter for our future work.

\section{References}

[1] Gold S., Rangarajan A.. Softmax to softassign: Neural network algorithms for combinatorial optimization Journal of Artificial Neural Networks. 1996;2:381-399.

[2] Rangarajan A.. Self-annealing and self-annihilation: unifying deterministic annealing and relaxation labeling Pattern Recognition. 2000;33:635-649.

[3] Guerrero F., Lozano S., Smith K.A., Canca D., Kwok T.. Manufacturing Cell Formation Using a New Self-organizing Neural Network Computers 83 Industrial Engineering. $2002 ; 42: 377-382$.

[4] Smith K.A., Palaniswami M., Krishnamoorthy M.. Neural Techniques for Combinatorial Optimization with Applications IEEE Transactions on Neural Networks. 1998;9:1301-1318.

[5] Rangarajan A., Gold S., Mjolsness E.. A Novel Optimizing Network Architecture with Applications Neural Computation. 1996;8:1041-1060. 
[6] Duch W., Itert L.. A posteriori corrections to classification methods in Neural Networks and Soft Computing (Rutkowski L., Kacprzyk J.. , eds.):406-411New York: Physica Verlag, Springer 2002.

[7] Tiňo P.. Equilibria of Iterative Softmax and Critical Temperatures for Intermittent Search in Self-Organizing Neural Networks Neural Computation. 2007;19:1056-1081.

[8] Kwok T., Smith K.A.. A Noisy Self-organizing Neural Network with Bifurcation Dynamics for Combinatorial Optimization IEEE Transactions on Neural Networks. 2004; $15: 84-88$.

[9] Agiza H.N.. Explicit Stability Zones for Cournot Game with 3 and 4 Competitors Chaos, Solitons and Fractals. 1998;9:1955-1966.

[10] Peng M.. Multiple bifurcations and periodic "bubbling" in a delay population model Chaos, Solitons and Fractals. 2004;25:1123-1130.

[11] Codreanu S., Laszlo M.. Some Considerations on the Bifurcation of the Fixed Point Generated by Iterated Function Systems Chaos, Solitons and Fractals. 1998;9:449$453(5)$.

[12] Bishop S.R., Sofroniou A., Shi P.. Symmetry-breaking in the response of the parametrically excited pendulum model Chaos, Solitons and Fractals. 2005;25:257-264.

[13] Sardanyés J.. Error threshold ghosts in a simple hypercycle with error prone selfreplication Chaos, Solitons and Fractals. 2008;in print. 